\title{
Long term effects of cocaine on the heart assessed by cardiovascular magnetic resonance at $3 \mathrm{~T}$
}

\author{
Alicia M Maceira', Carmen Ripoll², Juan Cosin-Sales ${ }^{3}$, Begoña Igual', Mirella Gavilan', Jose Salazar4, \\ Vicente Belloch ${ }^{1}$ and Dudley J Pennell ${ }^{5^{*}}$
}

\begin{abstract}
Background: Cocaine is an addictive, sympathomimetic drug with potentially lethal effects. The prevalence and features of cocaine cardiotoxicity are not well known. We aimed to assess these effects using a comprehensive cardiovascular magnetic resonance (CMR) protocol in a large group of asymptomatic cocaine users.

Methods: Consecutive ( $n=94,81$ males, $36.6 \pm 7$ years), non-selected, cocaine abusers were recruited and had a medical history, examination, ECG, blood test and CMR. The CMR study included measurement of left and right ventricular (LV, RV) dimensions and ejection fraction (EF), sequences for detection of myocardial oedema and late gadolinium enhancement (LGE). Images were compared to a cohort of healthy controls.

Results: Years of regular cocaine use were $13.9 \pm 9$. When compared to the age-matched healthy cohort, the cocaine abusers had increased LV end-systolic volume, LV mass index and RV end-systolic volume, with decreased LVEF and RVEF. No subject had myocardial oedema, but 30\% had myocardial LGE indicating myocardial damage.

Conclusions: CMR detected cardiovascular disease in $71 \%$ of this cohort of consecutive asymptomatic cocaine abusers and mean duration of abuse was related to probability of LV systolic dysfunction.
\end{abstract}

Keywords: Cocaine, Magnetic resonance, Heart failure

\section{Background}

Cocaine is a highly addictive sympathomimetic drug with potentially lethal effects $[1,2]$. The annual prevalence of cocaine use is $0.3 \%$ to $0.5 \%$ of the world population aged 15-64 [3], corresponding to roughly 18 million people. Standardised mortality ratios suggest that mortality is 4-8 times higher among cocaine users than age and gender peers in the general population [4].

Cocaine cardiotoxicity appears multifactorial through several mechanisms involving the development of ischemia and infarction [5], ventricular hypertrophy [6], systolic dysfunction [7], arrhythmias [8], endocarditis [9], and aortic pathology [10]. Cocaine is usually consumed along with other substances, mainly ethanol and tobacco, which can exacerbate the deleterious effects of cocaine [11].

The majority of studies that have assessed the prevalence and pattern of cardiovascular involvement due to

\footnotetext{
* Correspondence: d.pennell@ic.ac.uk

${ }^{5} \mathrm{NIHR}$ Cardiovascular Biomedical Research Unit, Royal Brompton Hospital \& Imperial College, London, UK

Full list of author information is available at the end of the article
}

cocaine abuse are autopsy series [12] or retrospective studies that focus on patients who presented with a particular disease or symptom in whom cocaine use was retrospectively evaluated. There are limited data on the effect of cocaine in asymptomatic users [13], and more evidence is needed. Moreover, the majority of the published studies have been done with echocardiography, include a modest sample size, and do not take into account pattern and time course of cocaine use and concomitant use of other substances.

Cardiovascular magnetic resonance (CMR) is the current gold standard technique for the assessment of ventricular dimensions and function, with high accuracy and reproducibility and reference values established for both the left [14] and right ventricles [15]. CMR can provide a comprehensive evaluation of the cardiovascular system and has a unique capability for tissue characterization, which has been applied to the assessment of several cardiomyopathies [16-18].

Therefore, we aimed to prospectively assess the presence and pattern of cardiovascular involvement due to 
cocaine abuse in consecutive, asymptomatic cocaine abusers (CA) with a comprehensive CMR protocol, adjusting for the effect of other concomitant variables such as tobacco or alcohol abuse.

\section{Methods}

This was a prospective study for which we recruited consecutive asymptomatic CA (males and females, 1860 years) who fulfilled the criteria for cocaine addiction or abuse [19], who were attending a rehabilitation clinic for the first time and who had no exclusion criteria (Table 1). The maximum time interval allowed between the last cocaine use and the CMR study was 3 months. All the eligible CA were informed about the study and invited to participate. The study was approved by the institutional ethics committee (IRB of Hospital Arnau de Vilanova, Valencia, Spain) and all patients gave their informed consent. As a reference for normality, we used data obtained in a group of gender and age matched healthy volunteers that have been previously published [14].

\section{Medical history}

Data from the patients' medical records were collected. Hypertension and dyslipidemia were defined according to the current guidelines [20,21]. Patients were classified as non-smoker, ex-smoker (1 month to three years after quitting smoking), and smoker. Overweight and obesity were defined as body mass index of $25-29.9 \mathrm{~kg} / \mathrm{m}^{2}$ and $\geq 30 \mathrm{~kg} / \mathrm{m}^{2}$, respectively. Diabetes mellitus was defined according to the ADA 2008 criteria [22]; from 2010 onwards HbA1C was also considered [23]. Family history of coronary heart disease and pharmacological treatment at the time of the study entry were investigated.

\section{Psychiatric evaluation}

Cocaine abuse or dependence was ascertained using the Structured Clinical Interview for Diagnostic and

\section{Table 1 Exclusion criteria}

\begin{tabular}{|c|c|}
\hline & $\begin{array}{l}\text { Diabetes mellitus, defined as plasma glucose }>126 \mathrm{mg} / \mathrm{dL} \text { or use of } \\
\text { antidiabetic drugs }\end{array}$ \\
\hline & $\begin{array}{l}\text { Severe chronic renal insufficiency (GFR }<30 \mathrm{~mL} / \mathrm{min} / 1.73 \mathrm{~m}^{2} \text { calculated } \\
\text { with the MDRD equation) }\end{array}$ \\
\hline • & AIDS diagnosis, conditions with short life-expectancy \\
\hline & Consumption of design drugs within the last 5 years* \\
\hline & Allergy to iodine or gadolinium based contrast agents \\
\hline & Claustrophobia \\
\hline & Metallic implants (eg pacemakers, defibrilators) \\
\hline & Acute psychotic attack \\
\hline
\end{tabular}

*Design drugs investigated were METH (methamphetamine, also called speed, crystal meth), MDA (3,4- methylenedioxyamphetamine, also known as love drug), MDMA (3,4-methylenedioxy-N-methylamphetamine, also known as ecstasy) and MDEA / MDE (3,4- methylenedioxy -N-ethylamphetamine, also called Eve).
Statistical Manual of Mental Disorders, Fourth Edition (DSM-IV) [24] and by documenting a cocaine-positive urine screening test at the time of enrolment. Lifetime use was assessed with the Life Severity Index for cocaine and we recorded the route of administration (nasal inhalation, smoked, intravenous), frequency of use during the three months prior to CMR, date of last cocaine use and years of regular cocaine use [25,26]. Finally, concomitant use of alcohol and tobacco were investigated with validated standardized scales [24,27]. Years of alcohol use, current number of units of standard drinks, smoking history as well as number of cigarettes were also recorded.

\section{Physical examination and laboratory tests}

These included recording of weight, height, heart rate, blood pressure and a cardiovascular routine evaluation. Blood sampling was performed for blood count, usCRP, fasting glucose levels, lipid profile, GOT, GPT, GGT and fibrinogen. Urine cocaine screening tests for the cocaine metabolite benzoylecgonine (Multicassette Drug Test, SureScreen Diagnostics Ltd, United Kingdom) were done. A rest electrocardiogram was obtained prior to the CMR scan.

\section{CMR protocol}

The study was done in a $3 \mathrm{~T}$ scanner (Achieva $3 \mathrm{~T} \mathrm{TX}$, Philips, The Netherlands). Images were obtained using a 32-channel surface array coil with multitransmit parallel radiofrequency transmission. Multislice dark blood and bright blood single shot sequences were acquired. High resolution balanced SSFP cine sequences with retrospective gating were obtained in the usual cardiac views as well as in an oblique sagittal plane of the aorta. Typical parameters were $\mathrm{TR}=3.2 \mathrm{~ms}, \mathrm{TE}=1.49 \mathrm{~ms}$, field of view $=360 \mathrm{~mm} \times 290 \mathrm{~mm}$, matrix size $=256 \times 224$ (inplane resolution $1.4 \mathrm{~mm} \times 1.3 \mathrm{~mm}$ ), slice thickness $7 \mathrm{~mm}$, slice gap $3 \mathrm{~mm}$, flip angle $30^{\circ}$, temporal resolution $28 \mathrm{~ms}, 40$ phases. STIR sequences in end-expiration were acquired in the same views (TR/TE/TI/ matrix/slice thickness $=1800 \mathrm{~ms} / 75 \mathrm{~ms} / 190 \mathrm{~ms} / 220 \times 180 / 7 \mathrm{~mm}$ ). High resolution transverse multislice T2 FSE sequences of the thoracic aorta were obtained (TR/TE/matrix/slice thickness/gap $=2000 \mathrm{~ms} / 85 \mathrm{~ms} / 340 \times 290 / 7 \mathrm{~mm} / 3 \mathrm{~mm})$.

In the first 48 subjects, dipyridamole $(0.84 \mathrm{mg} / \mathrm{Kg}$ in 6 minutes) stress myocardial perfusion images were acquired by using a saturation prepared gradient-echo sequence (TR/TE/flip angle/turbo factor/matrix/spatial resolution of $2.8 / 0.9 / 18^{\circ} / 59 / 128 \times 128 / 2.5 \times 2.5 \times 8 \mathrm{~mm}$ ), in three ventricular short-axis sections during gadolinium bolus administration $(0.1 \mathrm{mmol} / \mathrm{Kg})$. Images were also acquired at rest at the end of the protocol. In the subsequent 46 subjects the same myocardial perfusion sequence was acquired at rest. 
Finally, segmented inversion-recovery sequences (IRTFE) were acquired for detection of late gadolinium enhancement (LGE), starting at least 5 minutes after contrast administration, and in the same views as the functional study $(\mathrm{TR} / \mathrm{TE} / \mathrm{matrix} / \mathrm{segments}=6.2 \mathrm{~ms} / 3.1 \mathrm{~ms} /$ $240 \times 210 / 25$ ). The TI was adjusted to the value required to null the signal from the healthy myocardium. Whenever LGE was seen, the same sequence with swapped phaseencoding direction and different TI was repeated to rule out artifacts.

All the images were analysed with dedicated software (Medis, Leiden, The Netherlands). Analysis was carried out by two observers with at least 10 year experience in CMR. The following variables were quantified: left and right (LV, RV) ventricular end-diastolic (EDV) and end-systolic (ESV) volumes, LV mass, end-diastolic wall thickness, end-diastolic and end-systolic diameters, left atrial (LA) maximum anteroposterior diameter and area. Regional wall motion abnormalities and myocardial edema were investigated. Myocardial perfusion was visually assessed. Localization, pattern of transmural extent and quantification of LGE were obtained. Additionally, aortic and pulmonary trunk dimensions were measured and aortic plaques detected.

A control group of 80 healthy subjects (40 males, 40 females) of the same age deciles as the study subjects (20-60 years of age), belonging to a cohort whose normal values of reference have been previously published elsewhere [14,15], were used as a modelled reference for comparison. Since the healthy controls had been scanned at $1.5 \mathrm{~T}$, we first carried out a comparison test on 8 subjects in which we acquired SSFP cines at both $1.5 \mathrm{~T}$ and $3 \mathrm{~T}$, with the same sequence parameters that were eventually used, and in whom ventricular parameters were measured. We obtained a typical interstudy variability of $<4 \%$ for all these variables and none of the differences observed between both field strengths were statistically significant. We also demonstrated typical intraclass correlation coefficients for absolute agreement between both analysis softwares $>0.90$.

\section{Statistical analysis}

The data obtained were transferred into a database program SPSS 17.0 (IBM, USA). All the study variables were tested for normality with the Kolmogorov-Smirnov test. Continuous variables were expressed as mean \pm standard deviation, categorical variables were presented as percentages. Student-t test was used with the continuous Gaussian variables or, alternatively, the Wilcoxon test was employed. Qualitative variables were analyzed with the Chi-square test. Analysis of variance (ANOVA) was used whenever more than two levels of a variable were observed. Logistic regression using stepwise method was employed to analyze the odds ratio (OR) of the severity variables with significant results in the univariate analysis.

\section{Results}

Initially, $132 \mathrm{CA}$ were recruited. For some reasons (Figure 1) only 94 CA completed the whole protocol and were included in the study (13 females, age range $22-53$ yrs, $13.9 \pm 9$ yrs of addiction). The time interval between the last episode of cocaine uptake and CMR was $53 \pm 40$ days. Data on cocaine and alcohol abuse are presented in Table 2.

Table 3 shows the CA's biological characteristics and cardiovascular risk factors. No significant differences were found in height, weight and blood pressure between CA and controls. There were $14 \mathrm{CA}$ with no cardiovascular risk factors, and 66, 11 and 3 with 1, 2 and 3 risk factors, respectively. The most prevalent risk factor was current smoking. Only 2 hypertensive CA were receiving therapy with ACE inhibitors. None of the dyslipidemic CA complied with therapy. Laboratory tests are summarised in Table 4. Ultra-sensitive C-reactive protein (usCRP) was significantly elevated in $19 \%$ of CA, gammaglutamil transpeptidase (GGT) in $8.5 \%$ and fibrinogen in $7.5 \%$. In all cases, urine cocaine metabolites at the time of the scan were negative. Although CA were asymptomatic, 2 of them mentioned palpitations during cocaine use. On examination there were no significant findings except for elevated blood pressure in 4 subjects.

ECG abnormality was found in 44 CA (46.8\%), which minor repolarization abnormalities such as flat $\mathrm{T}$ waves or $<0.5 \mathrm{~mm} \mathrm{ST}$ depression in 26 patients (27\%), significant repolarization abnormalities in the inferior leads in $4 \mathrm{CA}$ and in frontal leads in another 3. ECG criteria of LV hypertrophy $(\mathrm{LVH})$ [28] were present in $7 \mathrm{CA}$. Finally, $7 \mathrm{CA}$ showed sinus bradycardia, $1 \mathrm{CA}$ had Mobitz 1 second degree AV block and another CA had a short PR interval.

\section{CMR findings}

Intraobserver and interobserver variability were assessed for LV and RV parameters in a subset of $15 \mathrm{CA}$. Intraobserver variability was below $4 \%$ for left ventricular parameters and below 5\% for right ventricular parameters. Typical interobserver variabilities were below $5 \%$ and $5.5 \%$ respectively.

The results of the CMR scans in the CA group were compared to those of the age-matched control group (Table 5). The LVESV was significantly enlarged (30 \pm 8 vs $\left.26 \pm 5 \mathrm{~mL} / \mathrm{m}^{2}, \mathrm{P}<0.01\right)$ and the $L V$ mass index was increased $\left(80 \pm 13\right.$ vs $\left.69 \pm 9 \mathrm{~g} / \mathrm{m}^{2}, \mathrm{P}<0.05\right)$ in the CA group. The relative wall mass was also significantly increased $(1.07 \pm 0.22$ vs $0.87 \pm 0.12, \mathrm{P}<0.01)$. No relation was found between blood pressure levels and LV mass. The LV ejection fraction (EF) was decreased (59 $\pm 6 \%$ vs $67 \pm 5 \%, \mathrm{P}<0.01)$. 


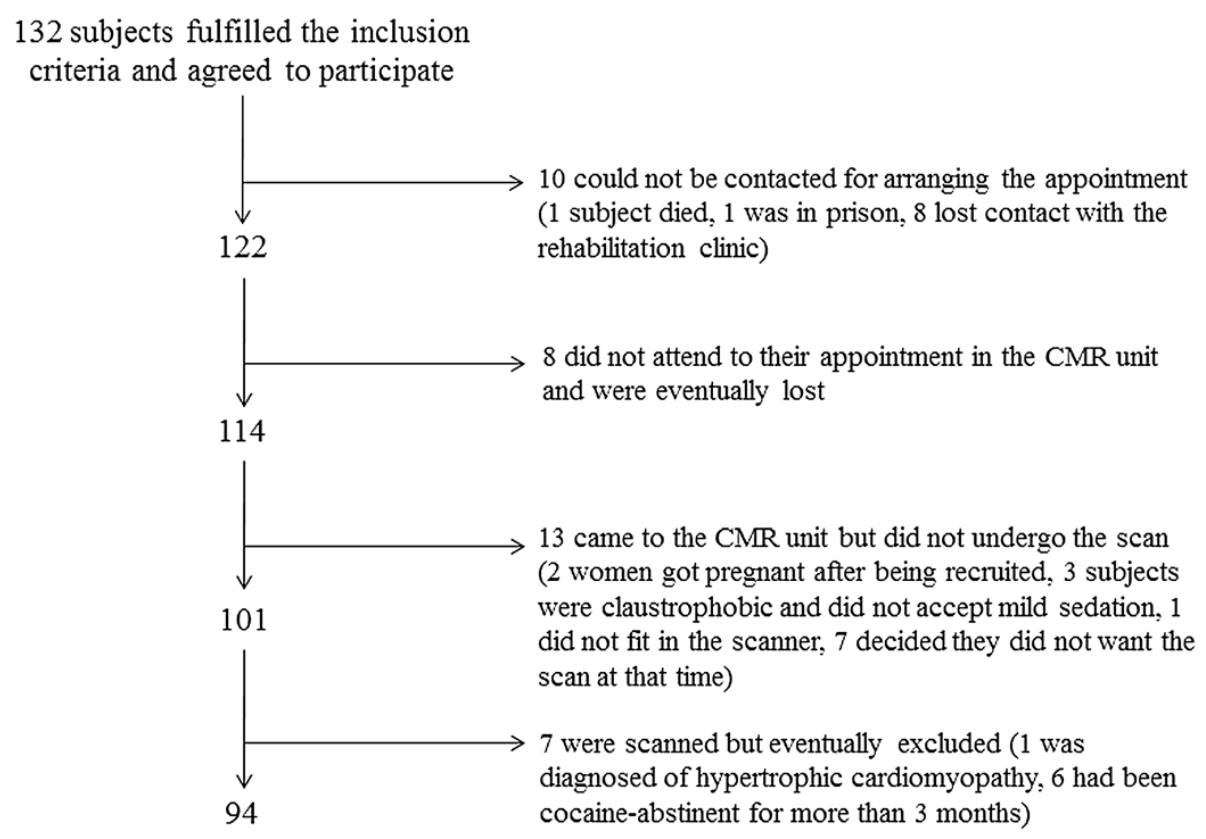

Figure 1 Patient recruitment and final participation in the study.

With regard to the RV, interestingly and similarly to the LV, the RVESV was significantly increased in the CA group ( $36 \pm 9$ vs $28 \pm 4 \mathrm{~mL} / \mathrm{m}^{2}, \mathrm{P}<0.01$ ). Accordingly, there was a significant decrease in RVEF (56 $\pm 5 \%$ vs $65 \pm 5 \%, \mathrm{P}<0.01)$.

\section{Table 2 Data on cocaine and alcohol use}

\begin{tabular}{|c|c|c|}
\hline \multirow[t]{11}{*}{ Cocaine } & Age at the time of first use, yrs & $22.7 \pm 6.7$ \\
\hline & Time between last use and CMR, days & $53 \pm 40$ \\
\hline & Frequency of use in the past (number of uses) & $3.2 \pm 1.8$ \\
\hline & $\begin{array}{l}\text { Frequency of use in the past } 3 \text { months } \\
\text { (uses per month) }\end{array}$ & $3.8 \pm 1.6$ \\
\hline & $\begin{array}{l}\text { Maximum frequency of use in lifetime } \\
\text { (uses per month) }\end{array}$ & $7.5 \pm 1.5$ \\
\hline & Amount of consumption in the past month $(\mathrm{g})$ & $3.8 \pm 1.6$ \\
\hline & Years of regular cocaine use (yrs) & $13.9 \pm 9$ \\
\hline & Route of administration & \\
\hline & Nasal insufflation & $81 \%$ \\
\hline & Smoked & $16 \%$ \\
\hline & Intravenous & $3 \%$ \\
\hline \multirow[t]{7}{*}{ Alcohol } & Subjects with alcohol abuse or dependence, $\mathrm{n}(\%)$ & $49(51 \%)$ \\
\hline & Age at the time of first use, yrs & $16 \pm 4$ \\
\hline & Time between last use and CMR (days) & $120 \pm 237$ \\
\hline & $\begin{array}{l}\text { Frequency of use in the past month } \\
\text { (number of uses) }\end{array}$ & $2.7 \pm 2.3$ \\
\hline & $\begin{array}{l}\text { Frequency of use in the past } 3 \text { months } \\
\text { (uses per month) }\end{array}$ & $2.8 \pm 2.3$ \\
\hline & Amount of use in the past month (gr) & $120 \pm 170$ \\
\hline & Years of alcohol use & $20 \pm 7$ yrs \\
\hline
\end{tabular}

In a per-subject analysis, compared to normal controls, 9 CA (10\%) had increased LVEDV and 29 CA (31\%) had an increased LVESV. Furthermore, 32 CA (34\%) showed decreased LVEF, the lowest being 48\% (Figure 2, Additional file 1: Video S1). Mild regional wall motion

Table 3 Biological data and cardiovascular risk factors

Males/females 81/13

Age (yrs)

$36.6 \pm 7$

Heart rate (bpm)

$71 \pm 12$

SBP $(\mathrm{mmHg})$

$127 \pm 10$

DBP $(\mathrm{mmHg})$

$82 \pm 11$

Weight $(\mathrm{Kg})$

$77 \pm 16$

Height $(\mathrm{cm})$

$173 \pm 7$

Body mass index $(\mathrm{Kg} / \mathrm{m} 2)$

$25.8 \pm 4.5$

Subjects with overweight, $\mathrm{n}(\%)$

$27(28.7 \%)$

Subjects with obesity, $\mathrm{n}(\%)$

$8(8.5 \%)$

Smoking habitus

Currently smoking, $\mathrm{n}(\%) \quad 71(75 \%)$

Ex-smoker, n (\%) $5(5.3 \%)$

Years of smoking habitus $\quad 13.5 \pm 9.1$

$\begin{array}{ll}\text { Cigarettes per day } & 13 \pm 7\end{array}$

Dyslipidaemia, n (\%) 10 (10.6\%)

Years of known dyslipidaemia $\quad 1.9 \pm 2.0$

Hypertension, $n(\%) \quad 4$ (4.2\%)

Years of known hypertension $\quad 3.6 \pm 6.4$

Family history of CAD, n (\%) 6 (6.3\%)

$S B P$ systolic blood pressure, $D B P$ diastolic blood pressure. 


\section{Table 4 Laboratory findings}

\begin{tabular}{ll}
\hline usCRP $(\mathrm{mg} / \mathrm{L})^{*}$ & $1.8(0.2,32)$ \\
subjects increased with usCRP $(\mathrm{n}, \%)$ & 18,19 \\
Glucose $(\mathrm{mg} / \mathrm{dL})$ & $79 \pm 18$ \\
Total cholesterol (mg/dL) & $250 \pm 42$ \\
LDL cholesterol (mg/dL) & $127 \pm 33$ \\
Triglycerides (mg/dL) & $244 \pm 140$ \\
Fibrinogen (mg/dL) & $475 \pm 45$ \\
Gammaglutamiltranspeptidase (IU/L) & $79 \pm 32$ \\
Fosfatase alkaline (IU/L) & $159 \pm 29$ \\
\hline
\end{tabular}

*usCRP is presented as median (minimum, maximum).

abnormalities were seen in $5 \mathrm{CA}$, which were located in all cases in the apical segments (Figure 3). With regard to LV morphology, 12 CA (13\%) showed concentric remodelling and 28 (30\%) showed LVH which was in 15 cases concentric (Additional file 2: Video S2) and in another 13 eccentric (Additional file 3: Video S3). LA dilatation was observed in $7 \mathrm{CA}$ (7.4\%), 4 of which had LVH and another 2 LV dilatation. With regard to the RV, 4 CA (4\%) had RVEDV above normal values, and as many as 12 (13\%) had an RVESV above the normal range. Finally, 16 CA (17\%) had decreased RVEF, with the lowest value being 43\% (Figure 2, Additional file 4: Video S4). Overall, 13 CA (14\%) had biventricular systolic dysfunction (Additional file 5: Video S5).

No CA showed increased myocardial signal intensity indicating oedema on STIR sequences.

In the first 48 subjects dipyridamole stress myocardial perfusion imaging was performed, we observed perfusion defects in 4 patients,

Table 5 Per-group analysis of CMR derived parameters

\begin{tabular}{llll}
\hline & Cocaine abusers & Controls & P \\
\hline LVEDVi $(\mathrm{mL} / \mathrm{m} 2)$ & $72 \pm 15$ & $76 \pm 9$ & NS \\
LVESVi $(\mathrm{mL} / \mathrm{m} 2)$ & $30 \pm 8$ & $26 \pm 5$ & $<0.01$ \\
LVSVi $(\mathrm{mL} / \mathrm{m} 2)$ & $42 \pm 9$ & $51 \pm 6$ & $<0.01$ \\
LVEF $(\%)$ & $59 \pm 5$ & $68 \pm 4$ & $<0.01$ \\
LVMi $(\mathrm{g} / \mathrm{m} 2)$ & $76 \pm 15$ & $69 \pm 4$ & 0.015 \\
RWM $(\mathrm{g} / \mathrm{mL})$ & $1.07 \pm 0.22$ & $0.87 \pm 0.12$ & $<0.01$ \\
RVEDVi $(\mathrm{mL} / \mathrm{m} 2)$ & $74 \pm 16$ & $79 \pm 11$ & NS \\
RVESVi $(\mathrm{mL} / \mathrm{m} 2)$ & $36 \pm 9$ & $28 \pm 4$ & $<0.01$ \\
RVSV $(\mathrm{mL} / \mathrm{m} 2)$ & $42 \pm 9$ & $51 \pm 7$ & $<0.001$ \\
RVEF $(\%)$ & $56 \pm 5$ & $65 \pm 5$ & $<0.001$ \\
LA AP diameter (cm) & $31.2 \pm 5.1$ & $30.8 \pm 4.8$ & NS \\
LA area (cm2) & $23.5 \pm 4.0$ & $24.7 \pm 4.0$ & NS \\
\hline
\end{tabular}

$L V$ left ventricle; $R V$ right ventricle; EDVi end-diastolic volume index, ESVi left ventricular end-systolic volume index, SVi stroke volume index, $E F$ left ventricular ejection fraction, $L V M i$ left ventricular mass index, $R W M$ relative wall mass, $L A$ : left atrium, $A P$ anteroposterior.
Dipyridamole stress myocardial perfusion images were visually analysed in the first $48 \mathrm{CA}$. Only 4 CA showed perfusion abnormalities, which in all cases were small, subendocardial, short-lasting and not suggestive of significant epicardial coronary artery disease [29]. These perfusion defects were associated with concentric LVH and concentric LV remodelling in $2 \mathrm{CA}$, respectively, but not with ventricular dilatation or systolic dysfunction. In the remaining $46 \mathrm{CA}$, no myocardial perfusion defects were observed at rest.

On LGE imaging, 29 CA (30\%) showed focal myocardial gadolinium enhancement. LGE extension was very limited, affecting 1 myocardial segment in $27 \mathrm{CA}$, and 2 segments in the remaining 2 CA. In 1 CA, LGE was subendocardial and located in the inferior wall, in another 2 CA a subepicardial pattern was found, it was midmyocardial in 14, and finally, it was located at the inferior $\mathrm{RV}$ insertion point in the remaining $10 \mathrm{CA}$. The mean percentage of focal myocardial fibrosis was $0.26 \pm 0.5 \%$ of LV mass (Figure 4). The presence or extent of LGE was not associated with the presence of regional wall motion abnormalities, LV hypertrophy or LV or RV dilatation or systolic dysfunction. Furthermore, the pattern of LGE of the inferior RV insertion point did not appear to be related to dilatation of either the RV or the pulmonary trunk.

Aortic and pulmonary trunk dimensions of CA were compared to published reference ranges [30,31]. Mean aortic sinus diameter was $32 \pm 3.5 \mathrm{~mm}$ (range $21-40 \mathrm{~mm}$ ), and mild dilatation of the sinus was observed in $9.6 \%$ of CA. Likewise, mean diameter of the ascending aorta was $28.7 \pm 4.2 \mathrm{~mm}$ (range $21-41 \mathrm{~mm}$ ), and $8.5 \%$ had dilatation of the ascending aorta. Mean diameter of the descending thoracic aorta was $21.5 \pm 2.9 \mathrm{~mm}$ (range $15-29 \mathrm{~mm}$ ) with $10.6 \%$ of CA showing dilatation of this segment. With regard to the pulmonary trunk, mean diameter at the bifurcation level was $22.7 \pm 3.1 \mathrm{~mm}$ (range $17-39 \mathrm{~mm}$ ), and it was shown to be dilated in $6.4 \%$ of CA. Finally, T2W FSE sequences also detected small, mural aortic plaques in 7 CA.

\section{CMR findings and cardiovascular risk factors, laboratory findings, ECG}

No differences were found in ventricular parameters according to the presence of cardiovascular risk factors, elevated usCRP, GGT or fibrinogen. Patients who smoked had an OR of $1.109(1.027,1.198)$ for developing aortic plaques. There was a poor correlation between CMR and ECG for detection of LVH. Only 3 patients with $\mathrm{LVH}$ on CMR had electrocardiographic criteria of $\mathrm{LVH}$, while 4 patients with electrocardiographic LVH had normal LV mass on CMR. The presence of ECG abnormalities was not associated with a higher percentage of regional wall motion abnormalities, 


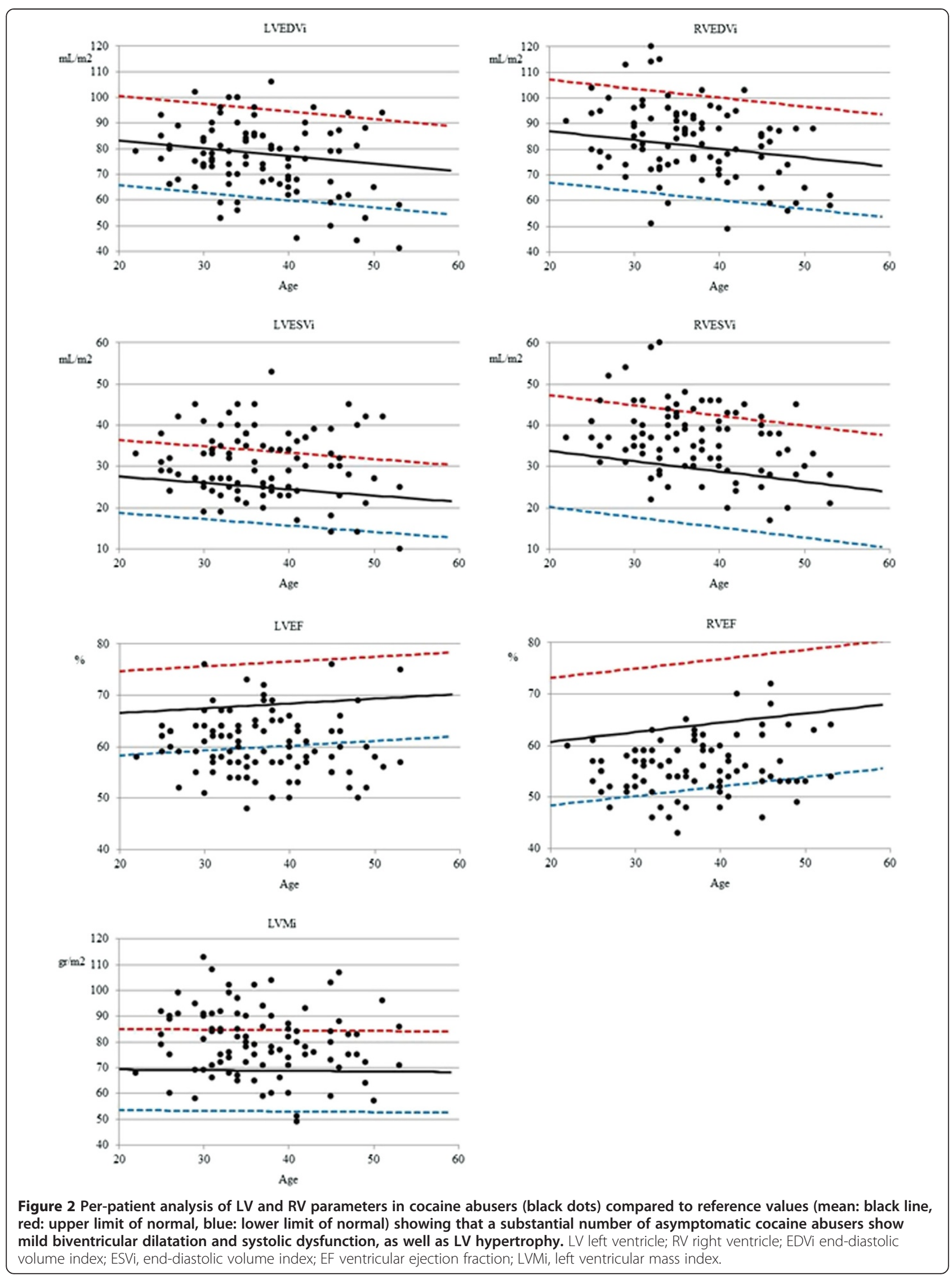



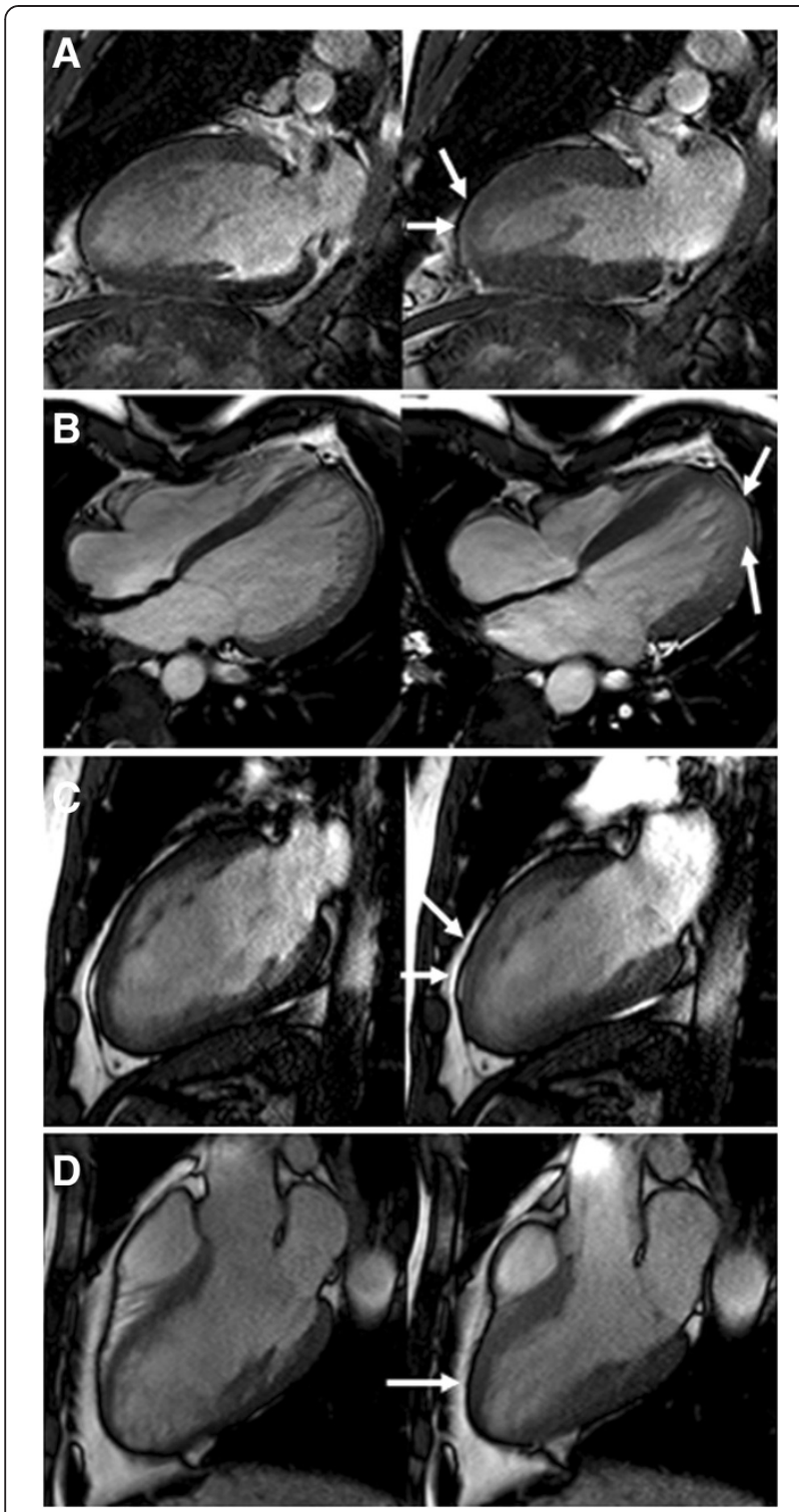

Figure 3 Regional wall motion abnormalities (arrows) were found in $\mathbf{4}$ subjects. A) and C) mild anteroapical hypokinesia; B) Apicolateral hypokinesia; D) distal septum hypokinesia.

LGE, LVH or dilatation, however there was a higher percentage of patients with LV systolic dysfunction among those with abnormal ECG findings $\left(\mathrm{X}^{2}=13.42, \mathrm{p}=0.009\right)$ (Figure 4).

\section{CMR findings and severity of cocaine abuse, way of use}

We selected the number of years of regular cocaine use as the most adequate variable to characterize the severity of cocaine abuse. This was tested against CMR findings shown as categorical variables. Only those variables with significant or nearly significant differences in univariable analysis were included in the multivariable analysis.
The numbers of years of regular cocaine use was significantly associated with the presence of LV systolic dysfunction $(\mathrm{P}<0.01)$ and with aortic dilatation $(\mathrm{P}<0.05)$, and borderline significantly for RV systolic dysfunction $(\mathrm{P}=0.06)$. Logistic regression showed that each year of regular cocaine use increased the risk of mild LV systolic dysfunction by nearly $10 \%(\mathrm{OR}=1.08,95 \% \mathrm{CI}=1.016-1.15$, $\mathrm{P}<0.01$ ). No significant differences were found in CMR findings between nasal, intravenous or smoked cocaine users. Likewise, no correlation was found between CMR findings and time since last use.

\section{CMR findings and concomitant alcohol abuse or dependence}

Among cocaine abusers, 51\% of subjects had concomitant alcohol abuse or dependence. No significant differences in ventricular dimensions and function were seen according to severity of alcohol consumption.

\section{Discussion}

We have used CMR at $3 \mathrm{~T}$ to describe the adverse effects caused by chronic cocaine consumption on the cardiovascular system in asymptomatic CA, compared to a group of healthy subjects, which consist mainly in mild LV dilatation, dysfunction and hypertrophy, RV dysfunction and focal myocardial fibrosis. Importantly, the RV was affected to some degree in nearly $25 \%$ of CA. Overall, some degree of cardiac involvement was shown in $71 \%$ of CA, and LV dysfunction was related to number of years of regular cocaine use, which may have practical importance in these patients' medical care. It has been previously shown that cocaine is cardiotoxic in both symptomatic [32] and asymptomatic $[13,33] \mathrm{CA}$, but, as far as we are aware, this is the first comprehensive study to assess with CMR cocaine cardiac and vascular toxicity in a reasonably large cohort of consecutive, non-selected, asymptomatic CA, comparing LV and $\mathrm{RV}$ results to healthy age and gender matched controls.

\section{Cocaine and the left ventricle}

We observed mild LV dilatation in $31 \%$ of the subjects included. This is in concordance with previous literature $[34,35]$. Whether this represents cocaine-related cardiomyopathy or ischemic cardiomyopathy remains unclear but in our series no relation was found between LV dilatation and ECG signs of necrosis, or LGE suggestive of previous myocardial necrosis. We also observed global LV systolic dysfunction in $34 \%$ of our series. This prevalence was higher than in previous echocardiographic reports on asymptomatic patients, in which it ranged from $0 \%$ to $14 \%[7,13,32,36]$. Still, we only found mild systolic dysfunction, with the lowest LVEF being 48\%, probably because we assessed the chronic effect of cocaine and not the acute phase effect, and also because our cocaine abusers were asymptomatic and likely to have less severe 

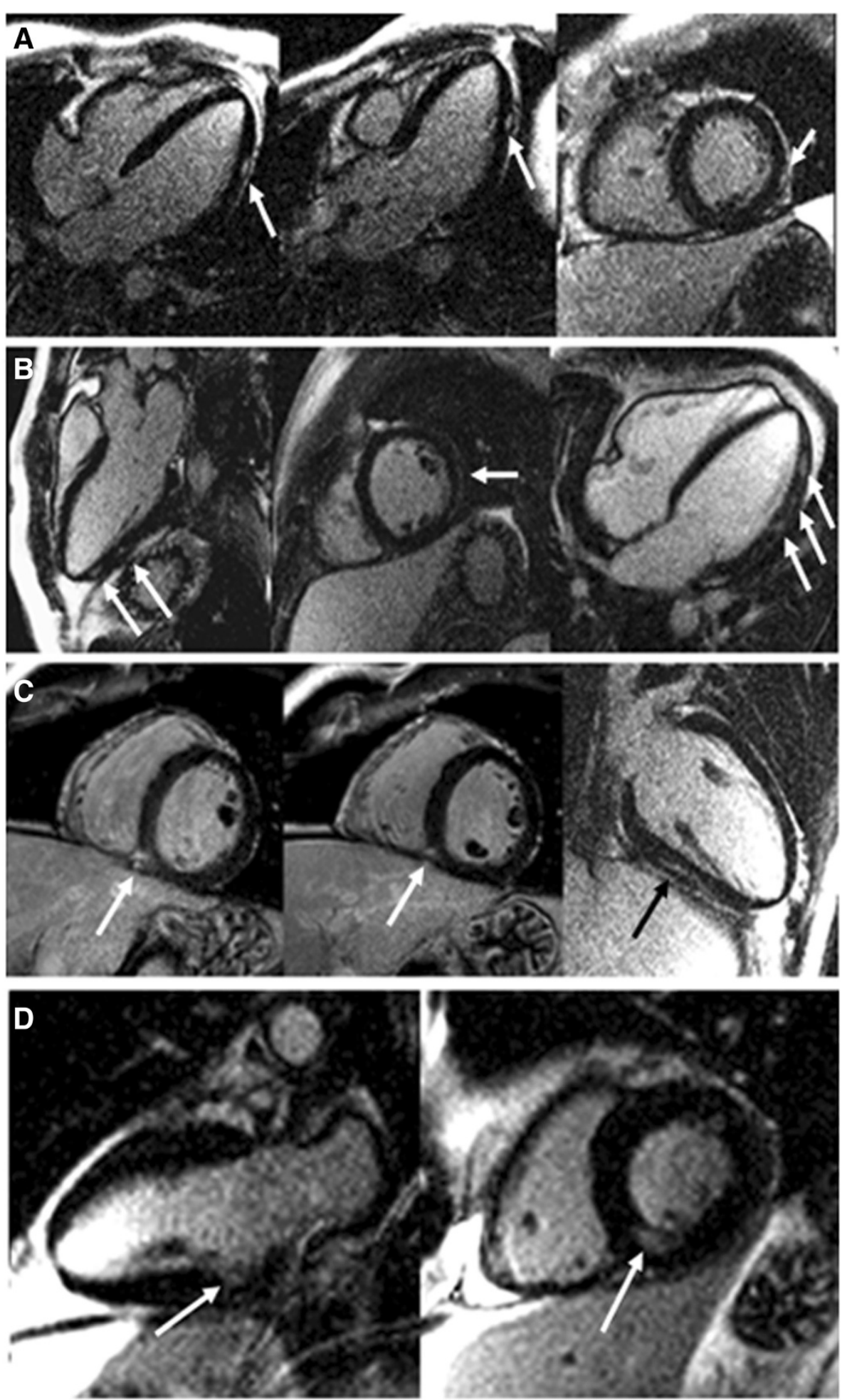

Figure 4 Late gadolinium enhancement (LGE) in 4 cocaine abusers (arrows). A) focal subepicardial LGE at the distal mid-lateral wall. B) several intramyocardial foci of LGE in the inferolateral and lateral wall. C) focal LGE in the mid inferior ventricular junction. D) limited subendocardial LGE in the basal inferior wall. 
cardiac involvement. With this study we cannot ascertain the mechanism leading to systolic dysfunction, as this was not our aim. Potential mechanisms have been suggested that could explain cocaine-related LV dysfunction including ischemia, repetitive sympathetic stimulation, direct toxicity, myocarditis, increased oxidative stress or activation of genes associated to apoptosis. We dare suggest that there is no single mechanism but several interacting, plus individual susceptibility.

With regard to LV anatomy, we found a pattern of concentric LV remodelling in $13 \%$ of subjects and LV hypertrophy in 30\%, which was in 15 cases concentric and in another 13 eccentric, not related to blood pressure levels. This finding may have prognostic value given the different prognosis that each pattern of LVH or remodeling has [37]. Autopsy-based studies assessing cocaine-related LVH usually include a small number of patients and use different reference ranges for diagnosing $\mathrm{LVH}$, so their results are hardly comparable. They usually show a high prevalence of $\mathrm{LVH}$, roughly around $50 \%$ [2,38-40], which might reflect a more advanced stage of the disease corresponding to autopsy-based studies, and a different method of measurement. In vivo echocardiographic studies have shown similar results ${ }^{6}$ but in asymptomatic abusers this had not been reported before. Unfortunately, so far no single mechanism has been proven to be responsible for LVH in this setting but some have been suggested, including the presence of frequent hypertensive peaks, direct stimulation of myocardial $\alpha$-adrenergic receptors, and increased expression of cardiomyocyte proteins mediated by protein kinase- $\mathrm{C}$ and calcium/calmodulin kinase II-dependent mechanisms.

\section{Cocaine and the right ventricle}

Another interesting finding in our study is the presence of RV involvement in these subjects. We observed RV dilatation in $13 \%$ of subjects. Likewise, RV systolic dysfunction was observed in $17 \%$ of subjects, which was in all cases global. Again, though the reason for this remains unclear, similar systemic mechanisms to those suggested for LV dysfunction could probably be applicable to the RV.

\section{Cocaine, myocardial oedema, perfusion and focal LGE}

We did not find signs of acute myocarditis such as increased focal myocardial signal intensity in the STIR sequences. In contrast to our findings, both autopsy [12,38] and clinical studies [13] have shown a significant proportion of myocarditis and oedema, associated with one or more positive urinary assay for cocaine metabolites, which probably indicates a more recent exposure to cocaine.

We could only assess stress myocardial perfusion in 48 CA. Still, we did not find any hypoperfusion pattern suggestive of significant epicardial coronary artery disease but only small, subendocardial, short-lasting perfusion defects in $4 \mathrm{CA}$, not related to ventricular dilatation or dysfunction in any case. Though invasive coronary angiography was not performed, we hypothesize that these perfusion defects could represent small vessel disease, which is a frequent finding in CA [2].

We found focal myocardial LGE in $30 \%$ of our patients. One patient showed a subendocardial pattern suggestive of myocardial necrosis, but not associated with a wall motion abnormality. In another two patients LGE was subepicardial, suggestive of past myocarditis. Finally, it was midmyocardial in 14 and in another 10 it was located in the inferior RV junction. The presence of coronary disease is not a sine qua non for the development of cardiac injury and late gadolinium enhancement. A previous publication in a casual cocaine user has demonstrated multiple spots of midmyocardial LGE taken as potential evidence of cocaine-induced vasoconstriction of coronary arterioles [41]. Furthermore, myocardial infarction can occur as a result of coronary thrombus in patients with otherwise normal coronary arteries [42]. Therefore, some of the midmyocardial areas of LGE found might well account for coronary vasospasm or small areas of thrombus occlusion, bearing in mind that this pattern is also observed in a number of cardiomyopathies.

Finally, we report the unexpected finding of LGE of the inferior RV insertion point in $10 \mathrm{CA}$. This particular pattern of LGE has been previously associated with adverse outcome in pulmonary hypertension, but we did not find RV wall motion abnormalities or pulmonary trunk dilatation in these patients, though this pattern was as frequent as midmyocardial LGE in patients with RV systolic dysfunction.

\section{Correlation of CMR findings with cardiovascular risk factors and ECG}

We observed ECG abnormalities of variable degree in nearly half of the subjects, similar to other authors [13,43-45], mainly consisting of minor changes. The presence of ECG changes regardless the type, was only associated to a higher proportion of LV systolic dysfunction. We also found a poor correlation between CMR and ECG for detection of LVH, concordant with previously acknowledged limitations of ECG in detecting LVH [46].

\section{CMR findings and severity of cocaine addiction, way of use}

Though it makes sense to quantify the amount of cocaine use as a marker of the severity and duration of cocaine abuse, there is no widely accepted quantitative variable. Cocaine abusers find it very difficult to recall accurately their usage. Also, as cocaine addiction may span many years, the abuse level can vary considerably. We chose number of years of regular cocaine use among a number of potential variables because this is simple for use in these patients, and also because it seemed the most accurate reflecting 
severity of cocaine abuse. We found that this variable was associated with the likelihood of cardiac involvement, reflecting cocaine cardiotoxicity, which may have practical utility for the overall medical care of these patients.

\section{CMR findings and concomitant alcohol abuse or dependence}

In our study, $51 \%$ of cocaine users had concomitant alcohol abuse or dependence. This proportion falls within the published ranges of $30-75 \%$ [47,48]. We did not find a relation between concomitant alcohol consumption and increased severity of cardiovascular involvement. This is in contrast with previously reported findings. When combined with ethanol, the concentration of cocaine increases, and the active metabolite cocaethylene is formed which blocks the reuptake of dopamine and helps to strengthen and prolong cocaine cardiovascular side effects [49]. It is not clear why alcohol did not have a further effect on cardiovascular findings in our study, but might arise from differences in our population characteristics and amount of alcohol consumption compared with previous studies.

\section{Study limitations}

Our study population may not be representative of subjects with only a recreational use of cocaine, or those who by their living standards have no financial problems maintaining their addiction who do not suffer economic, health or social problems, and thus may not seek medical care. Although we recruited consecutive patients, there is a potential selection bias as they were referred from the addictions treatment unit, which might indicate a more severe addiction or higher suspicion of heart disease. We cannot rule out coronary artery disease as a potential explanation to our findings as we did not perform relevant diagnostic investigations. We could not compare LGE findings in addicts with those of healthy subjects because we did not perform LGE in controls as that was not the scope of that particular study. Also, echocardiography was not performed so potential causes for RV dysfunction such as pulmonary hypertension could not be ruled out. Still, though $6 \%$ of addicts showed mild pulmonary trunk dilatation, we did not observe significant tricuspid regurgitation in the cine sequences in any of them. Finally, though the sample size of our study is one of the largest published to date, it remains fairly limited in power to detect small differences from normal. However, cocaine addicts are a difficult group of patients to study and are not reliable attenders for investigations and research.

\section{Conclusion}

CMR detected cardiovascular disease of variable degree in $71 \%$ of this cohort of consecutive cocaine abusers. The main findings were a decrease in systolic function of both left and right ventricles, an increase of left ventricular mass and the presence of focal fibrosis. There was a significant association between years of cocaine use and probability of LV systolic dysfunction.

\section{Additional files}

Additional file 1: Video S1. Cine sequence in the 4-chamber view of a CA showing mild left ventricular systolic dysfunction. LVEF was 49\%.

Additional file 2: Video S2. Cine sequence in the 4-chamber view of a CA with concentric LVH. LVMi was $96 \mathrm{~g} / \mathrm{m} 2$ and the relative wall mass was 1.18.

Additional file 3: Video S3. Cine sequence in the 4-chamber view of a CA with eccentric LVH. LVMi was $95 \mathrm{~g} / \mathrm{m} 2$ and the relative wall mass was 0.91 .

Additional file 4: Video S4. Cine sequence in the 4-chamber view of a CA showing right ventricular systolic dysfunction. RVEF was $44 \%$.

Additional file 5: Video S5. Cine sequence in the 4-chamber view of a CA showing mild biventricular systolic dysfunction. LVEF was $50 \%$ and RVEF was $46 \%$

\section{Competing interests}

The authors declare that they have no competing interests.

\section{Authors' contributions}

AMM: conception and design, data collection, analysis and interpretation of data, drafting of the manuscript, final approval. CR: conception and design, data collection, drafting of the manuscript, final approval. JC-S: design, critical review of the manuscript, final approval. Bl: design, data collection, critical review of the manuscript, final approval. MG: design, data collection, drafting of the manuscript, final approval. JS: conception and design, analysis and interpretation of data, critical review, final approval. VB: conception and design, critical review, final approval. DP: conception and design, interpretation of data, critical review of the manuscript, final approval.

\section{Acknowledgements}

This work was supported by the Consellería de Sanitat, Generalitat Valenciana. We thank Ms. Marisa Mocholi and Ms. Patricia Jorda for their helpful assistance during the recruiting and scanning processes.

\section{Funding}

This project has been partially founded by a research grant from the Consellería de Sanitat, Generalitat Valenciana, Spain.

\section{Author details}

${ }^{1}$ Cardiac Imaging Unit, ERESA Medical Center, Valencia, RIC, Spain.

${ }^{2}$ Addictions Treatment Unit of Campanar, La Fe Hospital, Valencia, Spain.

${ }^{3}$ Department of Cardiology, Hospital Arnau de Vilanova, Valencia, Spain. ${ }^{4}$ Department of Psychiatry, Consorcio Hospital General, Valencia, CIBERSAM, Spain. ${ }^{5} \mathrm{NIHR}$ Cardiovascular Biomedical Research Unit, Royal Brompton Hospital \& Imperial College, London, UK.

Received: 15 September 2013 Accepted: 24 March 2014 Published: 23 April 2014

\section{References}

1. Kloner RA, Rezkalla SH. Cocaine and the heart. N Engl J Med. 2003; 348:487-88.

2. Lucena J, Blanco M, Jurado C, Rico A, Salguero M, Vázquez R, Thiene G, Basso C. Cocaine-related sudden death: a prospective investigation in South-west Spain. Eur Heart J. 2010; 31:318-29.

3. UNODC. World Drug Report; 2012. United Nations publication, Sales No. E.12.XI.1.

4. Degenhardt L, Singleton J, Calabria B, McLaren J, Kerr T, Mehta S, Kirk G, Hall WD. Mortality among cocaine users: a systematic review of cohort studies. Drug Alcohol Depend. 2011; 113:88-95. 
5. Carrillo X, Curós A, Muga R, Serra J, Sanvisens A, Bayés-Genís A. Acute coronary syndrome and cocaine use: 8-year prevalence and inhospital outcomes. Eur Heart J. 2011; 32:1244-50.

6. Brickner ME, Willard JE, Eichhorn EJ, Black J, Grayburn PA. Left ventricular hypertrophy associated with chronic cocaine abuse. Circulation. 1991; 84:1130-35.

7. Diercks DB, Fonarow GC, Kirk JD, Jois-Bilowich P, Hollander JE, Weber JE, Wynne J, Mills RM, Yancy C, Peacock WF 4th. ADHERE scientific advisory committee and investigators. Illicit stimulant use in a United States heart failure population presenting to the emergency department (from the acute decompensated heart failure national registry emergency module). Am J Cardiol. 2008; 102:1216-19.

8. Magnano AR, Talathoti NB, Hallur R, Jurus DT, Dizon J, Holleran S, Bloomfield DM, Collins E, Garan H. Effect of acute cocaine administration on the QTc interval of habitual users. Am J Cardiol. 2006; 97:1244-46.

9. Chambers HF, Morris DL, Tauber MG, Modin G. Cocaine use and the risk for endocarditis in intravenous drug users. Ann Intern Med. 1987; 106:833-36.

10. Hsue PY, Salinas CL, Bolger AF, Benowitz NL, Waters DD. Acute aortic dissection related to crack cocaine. Circulation. 2002; 105:1592-95.

11. Baker J, Jatlow P, Pade P, Ramakrishnan V, McCance-Katz EF. Acute cocaine responses following cocaethylene infusion. Am J Drug Alcohol Abuse. 2007; 33:619-25

12. Pilgrim $\mathrm{L}$, Woodford $\mathrm{N}$, Drummer $\mathrm{OH}$. Cocaine in sudden and unexpected death: a review of 49 post-mortem cases. Forensic Sci Int 2012; 227:52-69.

13. Aquaro GD, Gabutti A, Meini M, Prontera C, Pasanisi E, Passino C, Emdin M, Lombardi M. Silent myocardial damage in cocaine addicts. Heart. 2011; 97:2056-62

14. Maceira AM, Prasad SK, Khan M, Pennell DJ. Normalized left ventricular systolic and diastolic function by steady state free precession cardiovascular magnetic resonance. J Cardiovasc Magn Reson. 2006; 8:417-26.

15. Maceira AM, Prasad SK, Khan M, Pennell DJ. Reference right ventricular systolic and diastolic function normalized to age, gender and body surface area from steady-state free precession cardiovascular magnetic resonance. Eur Heart J. 2006; 27:2879-88.

16. Mahrholdt H, Wagner A, Judd RM, Sechtem U, Kim RJ. Delayed enhancement cardiovascular magnetic resonance assessment of non-ischaemic cardiomyopathies. Eur Heart J. 2005; 26:1461-74.

17. Maceira AM, Joshi J, Prasad SK, Moon JCC, Perugini E, Harding I, Sheppard MN, Hawkins P, Pennell DJ. Cardiovascular magnetic resonance in cardiac amyloidosis. Circulation. 2005; 111:195-202.

18. Assomull RG, Prasad SK, Lyne J, Smith G, Burman ED, Khan M, Sheppard MN Poole-Wilson PA, Pennell DJ. Cardiovascular magnetic resonance, fibrosis and prognosis in dilated cardiomyopathy. J Am Coll Cardiol. 2006; 48:1977-85.

19. American Psychiatric Association. Diagnostic and statistical manual of mental disorders: DSM-IV [Internet]. 4th ed. Washington (DC): American Psychiatric Association; 1994. [cited 2010 Mar 8]. 866 p. Available from: http://dsm. psychiatryonline.org/signin.aspx.

20. Mancia G, De Backer G, Dominiczak A, Cifkova R, Fagard R, et al. Guidelines for the management of arterial hypertension: The Task Force for the Management of Arterial Hypertension of the European Society of Hypertension (ESH) and of the European Society of Cardiology (ESC). Eur Heart J. 2007; 28:1462-536.

21. Grundy SM, Cleeman Jl, Bairey CN, Brewer HB, Clark LT, Hunninghake D, Pasternak RC, Smith SC, Stone NJ. Implications of recent clinical trials for the national cholesterol education program adult treatment pnel III guidelines. Circulation. 2004; 110:227-39.

22. American Diabetes Association Position Statement. Standards of Medical Care in Diabetes -2008. Diabetes Care. 2008; 31:S12-54.

23. American Diabetes Association Position Statement. Standards of Medical Care in Diabetes -2010. Diabetes Care. 2010; 33:S11-61.

24. Sheehan DV, Lecrubier Y, Harnett Sheehan K, Janavs J, Weiller E, Keskiner A Schinka J, Knapp E, Sheehan MF, Dunbar GC. The validity of the Mini International Neuropsychiatric Interview (MINI) according to the SCID-P and its reliability. European Psychiatry. 1997; 12:232-41.

25. Hser YI, Shen H, Grella C, Anglin MD. Lifetime severity index for cocaine use disorder (LSI-cocaine): a predictor of treatment outcomes. J Nerv Ment Dis. 1999; 187:742-50.

26. Morales-Manrique C, Valderrama-Zurrián J, Castellano-Gómez M, Aleixandre-Benavent A. Exploratory factor analysis and validation of the lifetime severity index for cocaine, Spanish version (LSI-C-Spanish). J Nerv Ment Dis. 2007; 195:532-36.

27. Heatherton TF, Kozlowski LT, Frecker RC, Fagerström KO. The fagerström test for nicotine dependence: a revision of the fagerström tolerance questionnaire. Br J Addict. 1991; 86:1119-27.

28. Alfakih K, Walters K, Jones T, Ridgway J, Hall AS, Sivananthan M. New gender-specific partition values for ECG criteria of left ventricular hypertrophy: recalibration against cardiac MRI. Hypertension. 2004; 44:175-79.

29. Bernhardt P, Engels T, Levenson B, Haase K, Albrecht A, Strohm O. Prediction of necessity for coronary artery revascularization by adenosine contrast-enhanced magnetic resonance imaging. Int J Cardiol. 2006; 112:184-90.

30. Burman ED, Keegan J, Kilner PJ. Aortic root measurement by cardiovascular magnetic resonance: specification of planes and lines of measurement and corresponding normal values. Circ Cardiovasc Imag. 2008; 1:104-13.

31. Chuang ML, Gona P, Salton CJ, Tsao CW, Yeon SB, O'Donnell CJ, Manning WJ. Normal reference values for thoracic and abdominal aorta and main pulmonary artery dimensions by cardiovascular magnetic resonance: the framingham heart study. J Cardiovasc Magn Reson. 2013; 15(Suppl 1):256. Abstract.

32. Roldan CA, Aliabadi D, Crawford MH. Prevalence of heart disease in asymptomatic chronic cocaine users. Cardiology. 2001; 95:25-30.

33. Bertolet BD, Freund G, Martin CA, Perchalski DL, Williams CM, Pepine CJ. Unrecognized left ventricular dysfunction in an apparently healthy cocaine abuse population. Clin Cardiol. 1990; 13:323-28.

34. Willens HJ, Chakko SC, Kessler JM. Cardiovascular manifestations of cocaine abuse. A case of recurrent dilated cardiomyopathy. Chest. 1994; 106:594-600.

35. Arora S, Alfayoumi F, Srinivasan V. Transient left ventricular apical ballooning after cocaine use: is catecholamine cardiotoxicity the pathologic link? Mayo Clin Proc. 2006; 81:829-32.

36. Chakko S, Fernandez A, Mellman TA, Milanes FJ, Kessler KM, Myerburg RJ. Cardiac manifestations of cocaine abuse: a cross-sectional study of asymptomatic men with a history of long-term abuse of "crack" cocaine. J Am Coll Cardiol. 1992; 20:1168-74.

37. Ghali JK, Liao Y, Cooper RS. Influence of left ventricular geometric patterns of prognosis in patients with or without coronary artery disease. JACC. 1998; 31:1635-40.

38. Dressler FA, Malekzadeh S, Roberts WC. Quantitative analysis of amounts of coronary arterial narrowing in cocaine addicts. Am J Cardiol. 1990; 565:303-08

39. Virmani R, Robinowitz M, Smialek SE, Smyth DF. Cardiovascular effects of cocaine: an autopsy study of 40 patients. Am Heart J. 1988; 115:1068-76.

40. Karch SB, Stephens B, Ho CH. Relating cocaine blood concentratios to toxicity. An autopsy study of 99 cases. J Forensic Sci. 1998; 43:41-5.

41. Buchholz S, Grieve SM, Maher R, Figtree GA. Cocaine-induced myocardial injury seen as multiple mid-wall foci of late enhancement by contrastenhanced cardiac magnetic resonance imaging. Eur Heart J. 2010; 31:1422.

42. Robaei D, Grieve SM, Nelson GC, Bhindi R, Figtree GA. Cocaine-induced epicardial coronary artery thrombosis resulting in extensive myocardial injury assessed by cardiac magnetic resonance imaging. Eur Heart $\mathrm{J}$. 2010; 10:14

43. Schwartz BG, Rezkalla S, Kloner RA. Cardiovascular effects of cocaine. Circulation. 2010; 122:2558-69.

44. Hollander JE, Hoffman RS, Gennis P, Fairweather P, DiSano MJ, Schumb DA, Feldman JA, Fish SS, Dyer S, Wax P. Prospective multicenter evaluation of cocaine-associated chest pain. Cocaine Associated Chest Pain (COCHPA) Study Group. Acad Emerg Med. 1994; 1:330-39

45. Chakko S, Sepulveda S, Kessler KM, Sotomayor MC, Mash DC, Prineas RJ, Myerburg RJ. Frequency and type of electrocardiographic abnormalities in cocaine abusers (electrocardiogram in cocaine abuse). Am J Cardiol. 1994; 74:710-13.

46. Pewsner D, Jüni $P$, Egger M, Battaglia M, Sundström J, Bachmann LM. Accuracy of electrocardiography in diagnosis of left ventricular hypertrophy in arterial hypertension: systematic review. BMJ. 2007; 335:787. 
47. Blaho K, Logan B, Winbery S, Park L, Schwilke E. Blood cocaine and metabolite concentrations, clinical findings, and outcome of patients presenting to an ED. Am J Emerg Med. 2000; 18:593-98.

48. Brody SL, Slovis CM, Wrenn KD. Cocaine-related medical problems: consecutive series of 233 patients. Am J Med. 1990; 88:325-31.

49. Wilson $L D$, Malik $M$, Willson $H$. Cocaine and ethanol: combined effects on coronary artery blood flow and myocardial function in dogs. Acad Emerg Med. 2009; 16:646-55.

doi:10.1186/1532-429X-16-26

Cite this article as: Maceira et al: Long term effects of cocaine on the heart assessed by cardiovascular magnetic resonance at 3T. Journal of Cardiovascular Magnetic Resonance 2014 16:26.

\section{Submit your next manuscript to BioMed Central and take full advantage of:}

- Convenient online submission

- Thorough peer review

- No space constraints or color figure charges

- Immediate publication on acceptance

- Inclusion in PubMed, CAS, Scopus and Google Scholar

- Research which is freely available for redistribution 